\title{
Remodeling Capacity of Femoral Bone Defect by POP-CHA Bone Substitute: A Study in Rats' Osteoclast (First Series of POP-based Bone Graft Improvement)
}

\author{
Steven Kumar \\ Anne Handrini Dewi \\ Dyah Listyarifah \\ Ika Dewi Ana
}

Department of Dental Biomedical Sciences, Faculty of Dentistry, Gadjah Mada University, Yogyakarta 55281 Indonesia

E-mail: ikadewiana@ugm.ac.id

Received December 18, 2010; Accepted April 1, 2011

\begin{abstract}
Reconstruction of large bone defects caused by trauma, excision of tumors, and congenital malformations can be very difficult to perform. Bone engineering offers an option to improve bone reconstruction procedures. This interdisciplinary field applies the principles of biology and engineering to the development of functional substitutes for damaged bone. Our research aimed to find the ideal scaffold for bone regeneration, focusing on Calcium and Phosphate combination. In this study, Plaster of Paris (POP) was combined with $\mathrm{CHA}$ and implanted in femoral condyles of rats. According to the experimental result, it can be concluded that there was no significant difference in response to the implantation of POP and POP-CHA in Sprague Dawley rat femur condyle $(p<0.05)$. It can be stated that both POP-CHA and POP shows similar trait in bone healing.
\end{abstract}

Keywords: Plaster of Paris, carbonate apatite, osteoclast

\section{Introduction}

Reconstruction of large bone defects caused by trauma, excision of tumors, and congenital malformations can be very difficult to perform. It is usually associated with high complication rates. The areas which need mechanical pressure resistance need extremely solid bone substances for a good reconstruction. Standard procedure often involves bone transplantation (auto grafting). Generally, this procedure needs long term surgery with external fixation which can be hindered by complications due to mainly poor local tissue reaction, limitations caused by the amount of donor bone material and skin, the size of the defect and the healing process itself.

Another problem is donor site morbidity. Many complicationssuch asinfection, pain, loss of sensibility and hematoma have been described in this area ${ }^{1}$. It is not unusual that further surgical procedures have to take place due to these complications, often causing discomfort and disability².

Bone engineering offers an option to improve bone reconstruction procedures. This interdisciplinary field applies the principles of biology and engineering to the development of functional substitutes for damaged bone ${ }^{3}$. 
Our research aimed to find the ideal scaffold for bone regeneration, focusing on Calcium and Phosphate combination. In our previous study, we have been successful to apply dissolutionprecipitation method to provide macro-porous $\mathrm{CHA}$ (carbonated hydroxyapatite) bone substitute bodies from a combination of $\mathrm{CaSO}_{4} \cdot 1 / 2 \mathrm{H}_{2} \mathrm{O}$ and $\mathrm{Ca}(\mathrm{OH})_{2}$. The $\mathrm{CHA}$ resulted from the method is proven to be low crystalline $\mathrm{CHA}$ with $\mathrm{Ca} / \mathrm{P}$ ratio close to the one originated from the human bone ${ }^{4,5}$.

Regardless the superiority of the resulted materials, some complexities dealing with the valorization (scaling-up) of the method remains problems since we need to transform the $\mathrm{CaSO}_{4} \cdot 1 / 2 \mathrm{H}_{2} \mathrm{O}$ and $\mathrm{Ca}(\mathrm{OH})_{2}$ into $\mathrm{CHA}$. In view of this, we think of the possibility to provide materials which have ability to transform into CHA inside the body while providing osteo-conductivity and setting ability into critical bone defects.

On the other hands, calcium sulfate $\left(\mathrm{CaSO}_{4} \cdot 1 / 2 \mathrm{H}_{2} \mathrm{O}\right)$ or POP (Plaster of Paris) occupies a unique position in the universe of regenerative materials because it is well tolerated when used to fill bone defects and undergoes rapid and complete resorption without eliciting a significant inflammatory response ${ }^{6}$. When hemihydrate is mixed with water, the dihydrate is formed. This property makes it possible for POP to be set in situ when it is applied into critical bone defects. However, POP is also considered to be fast degradable material that allows complete resorption before bone defect area is completely covered by new bone formation.

In view of this, this study would like to introduce tissue engineering technology in which the local environment which is composed of scaffolds and bioactive molecules are combined to use for the natural induction of tissue regeneration ${ }^{3}$. If the combination of POP with osteoconductive and or osteoinductive materials, it will allow us to provide superb bone substitute candidate because the material will have good setting ability inside critical bone defects and have induction ability towards bone regeneration. It was considered in the first step the combination of POP-CHA.

The POP is relatively inexpensive and abundant in Indonesia while CHA can be produced in our laboratory by classical wet precipitation method. This study is considered to be the first series of the research on the improvement of POP-based bone graft by incorporation of molecules carrier hydrogel microsphere which is still on-going in our laboratory.

\section{Materials and Methods}

\section{Preparation of POP-based composite}

The CHA used in this study was prepared by wet precipitation method referred to the work of Leeuwenburgh and co-workers ${ }^{6}$. The preparation of carbonate hydroxyapatite ( $\mathrm{CHA}$ ) was started by using calcium hydroxide and phosphoric acid with $\mathrm{Ca} / \mathrm{P}$ was equivalent to 1.67 . Final dried substance from the reaction was taken out and thumped and made into thin powder.

Two bone grafts were prepared. The first, POP$\mathrm{CHA}$ was prepared by mixing POP and $\mathrm{CHA}$ powder in the ratio of 70:30 thus producing a combined POPCHA. The POP in other hand was mixed with distilled water $P: L=2: 1$ in ratio. Both of these materials which still in the state of powder were then put in a stainless steel mold $(3 \mathrm{~mm}$ in inner diameter by $3 \mathrm{~mm}$ thickness) and pressed uniaxially at $10 \mathrm{MPa}$ to prepare the composite. The composite was then put into $37^{\circ} \mathrm{C}$ incubator with $80 \%$ humidity for 24 hour to allow it to set completely. The set composite was sterilized using gamma irradiation done in National Atomic Research Institute (BATAN), Jakarta, Indonesia.

\section{Surgical procedure}

The animal protocol for this study was approved by Medical and Health Research Ethics Committee of The Faculty of Medicine, Gadjah Mada University. National Guideline for Animal Care was applied during the study. The subject of the study was 30 male 3 months old Sprague-Dawley rats with the weight ranging from 250 to 300 gram. Femoral condyles from both right and left leg of the rats were used for the implantation assigned randomly for 3 replicates per sub group. Surgery was performed 
under general anesthesia by intraperitoneal injection of ketamine hydrochloride. It was then left for 3 to 5 minutes for the anesthetic to come into effect and the rat to subdue. The part to be operated was shaved and skin was incised opened for a clear view of femoral condyles. After exposure of the bone surface, $2.5 \mathrm{~mm}$ holes were drilled. The holes were widened with a $3 \mathrm{~mm}$ twist drill to reach the final defect diameter of $3 \mathrm{~mm}$ with a depth of $3 \mathrm{~mm}$. The bone grafts which were prepared earlier were then substituted into the defect area. The operation site was irrigated using sterilized distilled water. Finally, the wounds were closed with vicryl 2.0 suture material. Antibiotics were administrated per oral as a safety measure preventing any bacterial infections.

\section{Histological observation}

Directly after retrieval on day $3,7,10,14$, and 28 , femoral condyles were fixed in $4 \%$ buffered formalin solution, dehydrated in a graded series of alcohol and embedded in paraffin. Hematoxylin- Eosine was used for staining the sections. Histological section of the slides was carried out using a light microscope in 6 observed views. The comparison of the bone graft namely POP-CHA and POP were done by observing and counting the number of osteoblast and osteoclast present after the implantation in Sprague Dawley rat femur condyle under a microscope up to 400X magnification with six sides of view were taken into account.

\section{Results and Discussion}

The study was carried out with the idea to compare two different bone grafts namely POP and POP-CHA and their effects on the physiological bone repairing mechanism. The effects were taken into record by observing the osteoblast and osteoclast cells as a parameter to determine not only the pace of bone regeneration or the remodeling process but also the quality of the mentioned bone grafts conveys in assisting the remodeling process. The pace of repair carries the meaning the time or period that it requires for bone regeneration while the quality of a bone graft simply means the ability to initiate and support the remodeling process for a rapid recovery.

With the above conceptual framework in mind, the study was carried out by using 30 rats (Sprague Dawley) as the subject where they were divided into two groups each with 15 rats. A defect of $3 \mathrm{~mm} \mathrm{X}$ $3 \mathrm{~mm}$ were inflicted on femur condyle of all the rats and subsequently one group was implanted with POP while the other with POP-CHA. These 2 groups were then divided into 5 sub groups in accordance with day 3 , day 7 , day 10 , day 14 and day 28 . Histological preparations of the femur condyle were then made and both osteoblast and osteoclast were observed and taken into record.

In order to eliminate subjective bias and enhance internal validity, the counting of osteoblast and osteoclast was also done by using double blind method. In this method, neither the observer nor the researcher has any clue on the day or the bone graft. Two observers observed and counted the osteoblast and osteoclast cells thus not only reducing bias but also error of perception. Subsequently correlation tests (Pearson Correlation) were done to describe the strength or the degree of relationship between two sets of data and in this case the data from the first and second observer on the numbers of cells present. The value of $r$ which is the degree of correlation has a higher relation between the two sets of data when it approaches +1 and has a poor relation when it approaches -1 .

According to the correlation test (Table 1 and 2) that was done in this study, the $r$ value on osteoblast and osteoclast count for POP is 0.988 and 1.000 respectively which means that the strength of relation between the two observers is high. Where else the $r$ value on the number of osteoblast and osteoclast for POP-CHA is 0.989 and 1.000 which interprets that both the observer are almost in total agreement. Since the data from both the observer has high correlation, thus it can be concluded that the data has high validity to the extent that it will be true to use one set of data from one observer for POP and POP-CHA to be analyzed. 
Table 1. The correlation of osteoblast and osteoclast for POP

\begin{tabular}{lll}
\hline & $1^{\text {st }}$ Observer & $2^{\text {nd }}$ Observer \\
\hline $1^{\text {st }}$ Observer of osteoblast & 1.000 & $0.988^{* *}$ \\
$2^{\text {nd }}$ Observer of Osteoblast & $0.988^{* *}$ & 1.000 \\
$1^{\text {st }}$ Observer of osteoclast & 1.000 & $1.000^{* *}$ \\
$2^{\text {nd }}$ Observer of Osteoclast & $1.000^{* *}$ & 1.000 \\
\hline & $* *$. Correlation is significant at the 0.01 level (2-tailed).
\end{tabular}

${ }^{* *}$. Correlation is significant at the 0.01 level (2-tailed).

Table 2. The correlation of osteoblast and osteoclast for POP-CHA

\begin{tabular}{lll}
\hline & $1^{\text {st }}$ Observer & $2^{\text {nd }}$ Observer \\
\hline $1^{\text {st }}$ Observer of osteoblast & 1.000 & $0.989^{* *}$ \\
$2^{\text {nd }}$ Observer of Osteoblast & $0.989^{* *}$ & 1.000 \\
$1^{\text {st }}$ Observer of osteoclast & 1.000 & $1.000^{* *}$ \\
$2^{\text {nd }}$ Observer of Osteoclast & $1.000^{* *}$ & 1.000 \\
\hline & $* *$ Correlation is significant at the 0.01 level (2-tailed).
\end{tabular}

Next the normality test was done to identify whether the data is in a normal distribution which is known to be bell shaped. The test result however showed that the data has a non significant value (sig.<0.05) for both osteoblast and osteoclast thus proving that the data is not normally distributed. Next homogeny test was done to discover should the variance in the groups have a same value. The result came as significant (sig.>0.05) which explains that the group has same variance value. However since the data is not normally distributed, non parametric tests were used for further analysis.

According to Table 3 , it is clear that POP-CHA had a jump start with a higher osteoblast count on day 3. It then declined and had almost the same amount of osteoblast count with POP for day 7 and day 10 but rose again sharply on day 14 before shying away on day 28.

Table 3. Number of osteoblast after implantation of POP and POP-CHA

\begin{tabular}{ccc}
\hline \multirow{2}{*}{ Duration (day) } & \multicolumn{2}{c}{ Number of Osteoblast (Mean \pm SD) } \\
\cline { 2 - 3 } & POP & POP-CHA \\
\hline 3 & $0.667 \pm 0.601$ & $3.940 \pm 1.760$ \\
7 & $5.778 \pm 3.513$ & $4.110 \pm 2.580$ \\
10 & $5.889 \pm 1.989$ & $5.830 \pm 2.404$ \\
14 & $8.777 \pm 1.080$ & $10.830 \pm 4.667$ \\
28 & $11.000 \pm 8.378$ & $3.440 \pm 0.788$
\end{tabular}


Kumar et al.

Table 4. The osteoclast count of POP and POP-CHA

\begin{tabular}{ccc}
\hline \multirow{2}{*}{ Duration (day) } & \multicolumn{2}{c}{ Number of Osteoclast (Mean \pm SD) } \\
\cline { 2 - 3 } & POP & POP-CHA \\
\hline 3 & $0 \pm 0$ & $0 \pm 0$ \\
7 & $0 \pm 0$ & $0 \pm 0$ \\
10 & $0.056 \pm 0.097$ & $0 \pm 0$ \\
14 & $0 \pm 0$ & $0 \pm 0$ \\
28 & $0 \pm 0$ & $0.056 \pm 0.097$ \\
\hline
\end{tabular}

The osteoclast count as shown in Table 4 has a rather poor cell count. The osteoclast was absent for the first few days in both POP and POP-CHA while it was present on day 10 for POP. For POP-CHA however the osteoclast was not in sight until day 28. Both osteoblast and osteoclast count for POP and POP-CHA that was obtained has been further analyzed to observe the significance of it. cells. It is then continued with Mann Whitney test which is also known as a non parametric test to compare two independent groups with sampled data. The Mann Whitney test was done to determine the significance between the groups.

The Kruskal Wallis test for POP group on the mean value of osteoblast shows a non significant value of 0.92 (sig.>0.05) while POP-CHA group also

Table 5. Kruskal Wallis test on osteoblast for POP and POP-CHA

\begin{tabular}{lcc}
\hline & POP & POP-CHA \\
\hline Chi-Square & 7.989 & 6.233 \\
Df & 4 & 4 \\
Asymp. Sig. & 0.920 & 0.182 \\
\hline
\end{tabular}

Table 6. Kruskal Wallis Test on Osteoclast for POP and POPCHA

\begin{tabular}{lcc}
\hline & POP & POP-CHA \\
\hline Chi-Square & 4.000 & 4.000 \\
Df & 4 & 4 \\
Asymp. Sig. & 0.406 & 0.406 \\
\hline
\end{tabular}

Subsequently, the Kruskal Wallis test was done to analyze the data. Kruskal Wallis method is known as a non-parametric test to compare 3 or more group with no assumption made on the distribution of the data. The Kruskal Wallis test was mainly done to identify whether the period of substitution has an effect on the number of osteoblast and osteoclast shows the same trait with a non significant value of 0.182 (sig.>0.05). The test for POP on the mean value of osteoclast also produced a non significant value of 0.406 (sig.>0.05) and the same goes for the mean value of osteoclast in POP-CHA with a non significant value of 0.406 (sig.>0.05). This shows that the substitution period has no clear effect on 
the number of osteoblast and osteoclast cells which notions that the bone graft namely POP and POP$\mathrm{CHA}$ has a same pace of regeneration process.

Proceeding with the Mann Whitney test to verify whether there is any significant difference between the groups, the test revealed that there was no significant (sig.>0.05) difference between all the groups. This interprets that the osteoblast and osteoclast cells between the groups showed a similar trait and thus giving an idea that the regeneration process were almost the same between the groups.

According to Čepelak and Čvorišćec ${ }^{7}$, the activity of osteoblast and osteoclast is a potential biochemical marker for bone formation. Thus it can be concluded that the comparison of both osteoblast and osteoclast cells reveals the success of a bone graft to initiate, support and assist the bone regeneration.

Initially, on day 3 both the group showed a similar trait where polimorphonuclear neutrophils (PMN) dominated the defect area. Although osteoblast were found in some areas but it was still scarce compare to PMN. Kalfas ${ }^{8}$ states that a bone healing process occurs in 3 stages, starting with inflammatory stage progressing to repair stage and finally the late remodeling stage. Thus it can be noted that on day 3 , the healing process is

still in the first stage which is inflammatory stage and the high number of $\mathrm{PMN}^{9}$, that the PMN is not only the most abundant class of white blood cells but also known as the first type of leukocyte recruited to sites of inflammation.

Subsequently on day 7 and 10, both PMN and osteoblast are present and could be seen on the defect area. This phenomenon is explained by Kalfas ${ }^{8}$, that the 3 distinct stages in bone healing could overlap. However, it could be seen that the number of PMN were dropping, while there were a steady increase in number of osteoblast in both groups. The osteoblasts can be seen on the surfaces of bone where active bone formations are occurring ${ }^{10}$. Thus it can be noted that the bone formation are underway in both groups. However on day 14, the number of osteoblast increased sharply. The sudden surge in the number of osteoblast is explained by $\mathrm{Kalfas}^{8}$, stating in a bone healing process, the first 2 weeks are the most critical period and in around $2^{\text {nd }}$ week is the time where mesenchymal cells differentiate into osteoblast and osteoclast.

However there was distinct difference on day 28 between the 2 groups where in POP the number of osteoblasts was still increasing while in POP-CHA the number of osteoblast dropped. The different trait seen on day 28 can be explained that in POP the increasing number of osteoblasts may reflect that the bone formation process is still active and underway however in POP-CHA the bone formation have entered the $3^{\text {rd }}$ stage which is the later remodeling stage. The low number osteoblasts in POP-CHA are due to the extracellular matrix which mineralize and embed the osteoblast cells within the matrix which also referred as osteocytes ${ }^{11}$.

The osteoclast however had a different story in POP and POP-CHA where in early stages of the bone healing process, the osteoclasts were not in sight. The osteoclasts were only recorded in POP on day 10 while in POP-CHA on day 28. The appearance of osteoclast at a later stage of the remodeling process is explained by Kalfas ${ }^{8}$ by stating the term 'creeping substitution' which carries the meaning that the osteoclastic resorption takes place slowly by resorbing the old necrotic bone which in other word could be time consuming.

Overall both POP and POP-CHA showed a good trait as to be a promising bone graft with a steady bone healing record. However when it is narrowed, the combination of POP and CHA showed an upper hand as a promising bone graft. The jump start that could be seen in POP-CHA could be due to the characteristic of both the materials.

According to Cabanas et $\mathrm{al}{ }^{12}$, the POP is one of the most biocompatible material and has a minimal inflammatory response when it is implanted in a bone defect. As such, with a minimal inflammation and a shorter duration of inflammatory stage, thus could initiate a faster bone healing process. On the other hand, as stated by Nilsson et al. ${ }^{13}$, the hydroxyapatite content in CHA is a biocompatible material with osteoconductive properties where it has shown to promote early bone in growth. These two theories prove that the POP-CHA could set off a quicker bone healing. 
Kumar et al.

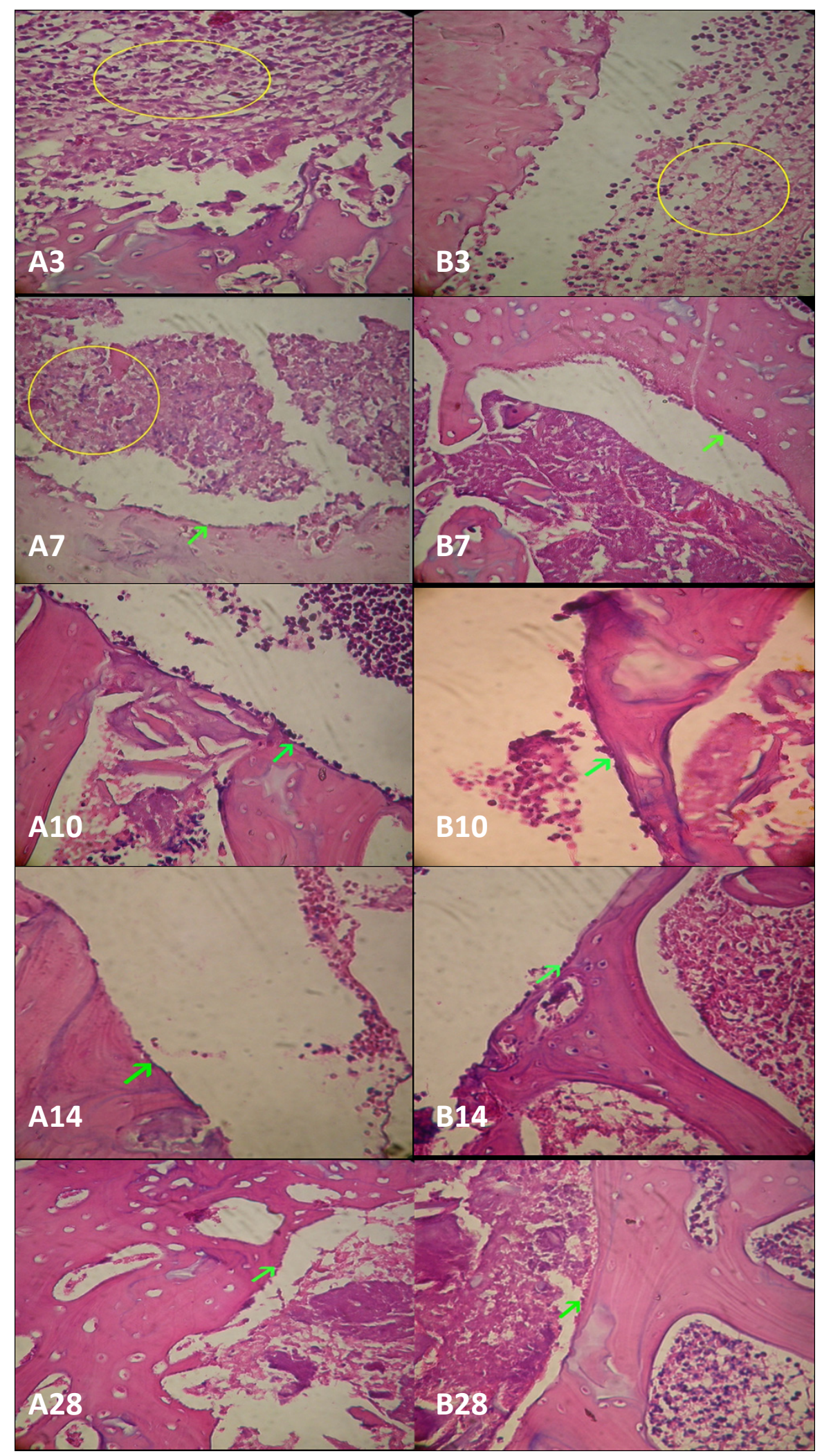

Figure 1. Histological image of POP (A) and POP-CHA (B) on day 3, 7, 10, 14, and 28 after implantations. Polymorphonuclear neutrophil is shown by yellow circle and osteoblast lining cells are shown by green arrow. 